\title{
BJMHR
}

British Journal of Medical and Health Research

Journal home page: www.bjmhr.com

\section{Health Related Quality of Life and Depression among Women with Poly Cystic Ovary Syndrome (PCOS) in Pakistan.}

\section{Madeeha Malik $^{1^{*}}$, Fazziya Latif ${ }^{2}$, Azhar Hussain $^{3}$ \\ 1. Hamdard Institute of Pharmaceutical Sciences, Hamdard University Islamabad, Pakistan}

\section{ABSTRACT}

Polycystic ovary syndrome is a multifactorial disease caused by both genetic and environmental factors acting concurrently with rising prevalence at an alarming rate. It is a health problem that affects 1 out of 10 women of childbearing age. PCOS have negative impact on quality of life resulting in depression. The present study was designed to assess health related quality of life and depression among polycystic ovary syndrome patients in Pakistan. A descriptive cross sectional study design was used to assess health related quality of life and depression among 152 women with PCOS diagnosed by Rotterdam criteria using PCOSQ-50 and HADS. Data was collected and statistically analysed. Significant difference $(\mathrm{p} \geq 0.05)$ among different domain of HRQoL and level of depression among women with PCOS was observed. Unmarried patients had better emotions while body hairs, weight and infertility effect married women. Rural patients had relatively poor HRQoL. Women with PCOS in age group 26-35yrs had impaired HRQoL due to infertility, emotions and increased weight. Obese patients with PCOS had relatively more impaired HRQoL as all domains were affected in them. Women with PCOS residing in rural areas were found relatively more depressed and those belonging to low educational and income background were comparatively found more depressed. The results of the present study concluded that polycystic ovary syndrome had a negative impact on health related quality of life of patients across all domains with a significant likelihood of depression. While all domains were affected, the greatest impact was seen on infertility followed by body hairs and role limitation due to increased psychological and emotional problems. Low educational and income background had negative effect on mental and emotional HRQoL of polycystic ovary syndrome patients and depression. More attention should be given on improvement of health related quality of life among PCOS patients to decrease the rate of treatment failure and improve treatment response.

Keywords: Depression, health related quality of life, poly cystic ovary syndrome (PCOS), women, Pakistan.

Please cite this article as: Malik M et al., Health Related Quality of Life and Depression among Women with Poly Cystic Ovary Syndrome (PCOS) in Pakistan. British Journal of Medical and Health Research 2020. 


\section{INTRODUCTION}

Polycystic ovary syndrome (PCOS) is a multifactorial disease caused by both genetic and environmental factors acting concurrently with rising prevalence at an alarming rate. It is a health problem that affects 1 out of 10 women of childbearing age. Approximately $6.6 \%$ of unselected reproductive-aged women are diagnosed with PCOS in USA. The annual cost burden is 93 million dollar for initial evaluation only and total cost for evaluation and treatment for reproductive-aged PCOS women is 4.36 billion dollar in USA. Polycystic ovary syndrome affects $15-20 \%$ of reproductive women ${ }^{1}$. Approximately $5-10 \%$ women are affected by PCOS in fertility ages ${ }^{2}$. PCOS have negative impact on quality of life. Infertility is main cause of decrease quality of life in women age above 25 year $^{3}$. PCOS can also lead to abortion ${ }^{2}$. Quality of life determinants depends upon different demographic variables. Hirsutism, oligomenorrhea or amenorrhea, hypertension, weight gain, hair loss and acne shows significant association with PCOS 4. Hirsutism is main cause of decrease quality of life among women below 25 years of age ${ }^{3}$. On the other hand, infertility is one of the main reason for decreased quality of life in women age above 25 year $^{3}$. The increasing prevalence of depression in PCOS patients has been reported significantly with time ${ }^{5}$. Obesity, body hair, and infertility may decrease selfconfidence and create depressive symptoms in patients with PCOS ${ }^{6}$. In addition, changes in hormonal levels may lead to anxiety directly ${ }^{6}$.Insulin resistance is significantly associated with depression in patients with polycystic ovary syndrome patients ${ }^{7}$. Poor body image cause inauspicious consequences on sexual satisfaction of women with PCOS ${ }^{8}$. Acne appearing mostly on face effects the personality of a patient which decreased quality of life ${ }^{8}$. Moreover, outer appearance cause depression in PCOS women ${ }^{9}$.

The importance of health related quality of life of polycystic ovary syndrome patients has been long recognized in developed countries but in developing countries like Pakistan this concept is still in early stages. The prevalence of PCOS among general population is 3-7 \% while in $20.7 \%$ of the cases it is associated due to different co morbidities and in $6-8 \%$ of the cases it leads to infertility ${ }^{10}$. PCOS is associated with obesity in $60 \%$ of the women and also increases the risk of diabetes and cardiovascular disease in $10 \%$ and $33 \%$ of the women, respectively ${ }^{10}$. A study reported that nearly $85-90 \%$ of women suffer oligomenorrhoea while $30-40 \%$ present with amennorhoea as clinical features of anovulation but not all patients show these symptoms while PCOS was diagnosed in $16 \%$ of the patient during surgery ${ }^{11}$. The prevalence of PCOS among women in Pakistan was reported to be $5-10 \%$ of those in fertility age while $40 \%$ of the women suffer from depression particularly in adolescent age ${ }^{12}$. Depression was found significantly associated with PCOS women in Pakistan ${ }^{4}$. Depression in PCOS patients was found mainly due to obesity, infertility, hirsutism, menstrual irregularity and insulin 
resistance ${ }^{13}$. Hirsutism was reported in $36.7 \%$ of the women with PCOS in Pakistan ${ }^{14}$. While almost $50 \%$ of patients with PCOS are obese ${ }^{11}$.

Although, polycystic ovary syndrome is a common endocrine disorder but only $10 \%$ of the population is aware of the disease in Pakistan ${ }^{14}$. Out of $51.8 \%$ obese women and $22.2 \%$ of them who were extremely obese, $55.6 \%$ were unaware of polycystic ovary disease ${ }^{15}$. The major reasons for the increasing burden of the disease is lack of awareness, healthcare facilities, technical equipment's for diagnosis and financial support due to which most of the people are left untreated, resulting in decrease quality of life and increased depression. In the past few decades, various contributing factors effecting quality of life were studied worldwide for better understanding of different aspects associated with decreased health related quality of life and increased psychosocial burden among PCOS women, but women reproductive health is still one of the neglected areas in most of the developing countries including Pakistan. Therefore, the present study was designed to assess health related quality of life and depression among polycystic ovary syndrome patients in Pakistan.

\section{MATERIALS AND METHOD}

A descriptive cross sectional study design was used to determine the health related quality of life and depression among polycystic ovary syndrome patients in Pakistan. Study approval was taken from the Ethical Committee of Hamdard University (BASR-81-5.2). Approval was also taken from Medical superintendents of different hospitals located in Rawalpindi and Islamabad. Patients were briefed regarding nature and objectives of the study and verbal and written consent were obtained prior to data collection. Respondents were assured of the confidentiality of their responses and their right to withdraw from the study at any time. Health care facilities both from public and private sector were included in study. Polycystic ovary syndrome patients diagnosed using Rotterdam criteria from different public and private clinics of Pakistan were included as study respondents. Rotterdam criteria require two of three features: polycystic ovaries (PCO) on ultrasound, an ovulatory irregular cycles or hyper androgenism. Polycystic ovary syndrome patients were selected on following criteria from age group of 15 to 50 years old, married or unmarried and persons who could speak \& understand Urdu or English. Any patient having known depression was excluded from the study. Women with ovulatory dysfunction from other causes such as hyperprolactinemia, as well as pregnancy in reproductive-aged women were also excluded.

Sample size was calculated using Raosoft ${ }^{\circledR}$ sample size calculator which came to be 323 patients in order to achieve 95\% confidence level with 5\% margin of error. As no updated list of number of polycystic ovary syndrome patients in Pakistan is available, convenient sampling technique was used. According to convenient sampling technique all the accessible respondents 
that were present at the time of data collection were included in study. The questionnaires were filled by 152 patients and the response rate of the study was $47 \%$. Two pre- validated questionnaires were used i.e. PCOS-Q (Polycystic Ovary Syndrome Questionnaire) to measure Health Related Quality of Life (HRQoL) and HADS (Hospital Anxiety \& Depression Scale) to measure depression.

The PCOSQ-50 is used worldwide to measure Health Related Quality of Life of polycystic ovary syndrome patients. It consist of 50 items representing 6 sections, namely; psychosocial and emotional, body weight and menstrual disorders, fertility, sexual function, body hair disorders, and coping. Each item could be answered by picking options on a 5-point Likert scale and will assess by a 7-point scale (1-poor/7no problem). The higher the scores, better the condition ${ }^{1}$. By using confirmatory factor analysis 43 items of PCOSQ show relevant validity and reliability to assess HRQoL ${ }^{8}$. The HADS Questionnaire consists of fourteen items designed to assess anxiety and depression in medical patients. Out of all, seven items are related to anxiety and seven to depression. Scores for each subscale (anxiety and depression) range from 0 to 21 with scores categorized as; Normal 0-7, mild 8-10, moderate 11-14, and severe 15-21. Scores for the entire scale (emotional distress) range from 0 to 42 , with higher scores indicate more distress.

Pilot testing was performed on $10 \%$ of sample size for reliability of tool. The value of Cronbach's alpha was 0.72 for Polycystic Ovary Syndrome Questionnaire (PCOS-50) and 0.75 for Hospital Anxiety and Depression Scale (HADS). The questionnaires were selfadministered to the respondents by principal investigator and collected back on the same day to avoid study biasness. After data collection, data was cleaned, coded and entered in SPSS version-21. Descriptive statistics comprising of percentages and frequencies were calculated. Mann-Whitney and Kruskal-Wallis Test $(\mathrm{p} \geq 0.05)$ were used to find the difference among different variables.

\section{RESULTS AND DISCUSSION}

Out of 152 respondents, $50.7 \%(n=77)$ were married, $46.1 \%(n=70)$ were unmarried, $2.6 \%$ $(n=4)$ divorced and $0.7 \%(n=1)$ was widow. Of the total respondents, 3.9\% $(n=6)$ were illiterate and $32.2 \%(n=49)$ were graduates. Regarding the job status of the respondents, $21.7 \%(n=33)$ were employed where as $78.3 \%(n=119)$ were unemployed, of whom 50\% $(n=76)$ were housewives and $28.3 \%(n=43)$ were studying. Out of all the respondents, $10.5 \%(n=16)$ had regular periods, $88.8 \%(\mathrm{n}=135)$ had irregular periods and $0.7 \%(\mathrm{n}=1)$ had no periods because of menopause. Moreover, 75.7\% ( $n=115)$ had no children and 2\% $(n=3)$ had more than four children. While, $33.6 \%(\mathrm{n}=51)$ were on hormonal therapy and $59.9 \%(\mathrm{n}=91)$ were not on any therapy. A detailed description is given (Table 1). 
Table 1: Demographic Characteristics of Respondents

\begin{tabular}{|c|c|c|c|c|c|}
\hline Variable & Indicator & Total n (\%) & Variable & Indicator & Total n (\%) \\
\hline \multirow[t]{4}{*}{ Age } & $15-25$ & $84(55.3)$ & \multirow{4}{*}{$\begin{array}{l}\text { Marital } \\
\text { status }\end{array}$} & Married & $77(50.7)$ \\
\hline & $26-35$ & $51(33.6)$ & & Single & $70(46)$ \\
\hline & \multirow[t]{2}{*}{$36-50$} & \multirow[t]{2}{*}{$17(11.2)$} & & Divorced & $4(2.6)$ \\
\hline & & & & widow & $1(0.7)$ \\
\hline \multirow[t]{6}{*}{ Qualification } & Illiterate & $6(3.9)$ & & $\leq 15,000$ & $46(30.3)$ \\
\hline & Primary & $25(16.4)$ & \multirow{5}{*}{ level } & $15,001-25,000$ & $32(21.1)$ \\
\hline & Secondary & $55(36.2)$ & & $25,001-35,000$ & $41(27)$ \\
\hline & Bachelors & $49(32.2)$ & & $35,001-50,000$ & $26(17)$ \\
\hline & Masters & $16(10.5)$ & & $\geq 50,000$ & $7(4.6)$ \\
\hline & Post graduate & $1(0.7)$ & & & \\
\hline \multirow[t]{6}{*}{ Working status } & Employed & $33(21.7)$ & \multirow{6}{*}{$\begin{array}{l}\text { No. of } \\
\text { children }\end{array}$} & None & $115(75.6)$ \\
\hline & House wife & $76(50)$ & & one & $10(6.6)$ \\
\hline & studying & $43(28.3)$ & & two & $10(6.6)$ \\
\hline & & & & three & $11(7.2)$ \\
\hline & & & & four & $3(2)$ \\
\hline & & & & $>$ four & $3(2)$ \\
\hline \multirow{4}{*}{$\begin{array}{l}\text { Type of } \\
\text { comorbidity }\end{array}$} & None & $134(88.2)$ & \multirow{4}{*}{$\begin{array}{l}\text { Type of } \\
\text { therapy }\end{array}$} & Hormonal therapy & $51(33.8)$ \\
\hline & Hypertension & $9(5.9)$ & & Other & $10(6.6)$ \\
\hline & Other & $2(1.3)$ & & None & $91(59.9)$ \\
\hline & Both & $7(4.6)$ & & & \\
\hline \multirow{3}{*}{$\begin{array}{l}\text { Thyroid } \\
\text { problem }\end{array}$} & Yes & $3(2)$ & \multirow{3}{*}{$\begin{array}{l}\text { Built of } \\
\text { patient }\end{array}$} & Heavy & $87(57.2)$ \\
\hline & No & $149(98)$ & & Normal & $37(24.4)$ \\
\hline & & & & slim & $28(18.4)$ \\
\hline \multirow{3}{*}{$\begin{array}{l}\text { Problem } \\
\text { conceiving }\end{array}$} & Yes & $54(35.5)$ & \multirow[t]{3}{*}{ periods } & Regular & $16(10.5)$ \\
\hline & no & $98(64.5)$ & & Irregular & $135(88.8)$ \\
\hline & & & & No periods & $1(0.7)$ \\
\hline
\end{tabular}

The results showed lowest scores for HRQoL were observed in domain of infertility $(38.03, \pm$ $27.820)$ followed by domain of psychosocial and emotional $(46.98, \pm 22.51)$ whereas highest scores were observed in the domain of body weight $(53.36, \pm 21.59)$. A detailed description is given in (Table 2).

Table 2 Domains of Health Related Quality of Life (HRQOL) among PCOS Women

\begin{tabular}{lccc}
\hline Indicator & Mean & Median & Standard Deviation \\
\hline Psychosocial and emotional & 46.98 & 45.00 & 22.515 \\
Infertility & 38.03 & 31.25 & 27.820 \\
Menstrual & 48.73 & 46.43 & 24.456 \\
Body weight & 53.36 & 50.00 & 21.598 \\
Body hair & 47.70 & 45.00 & 22.507 \\
\hline
\end{tabular}

Comparison of HRQoL domains in relation to different demographic characteristics demonstrated significant difference $(\mathrm{p} \geq 0.05)$ among different domain of HRQoL. The comparison showed that unmarried patients had better emotions while body hairs, weight and infertility effect married women. Rural patients had relatively poor HRQoL. Emotions were better managed among women with PCOS in age group 36-50 yrs while body weight, body hairs and infertility were found better among women of age group 15-25yrs while women with 
PCOS in age group 26-35yrs had impaired HRQoL due to infertility, emotions and increased weight. Obese patients with PCOS had relatively more impaired HRQoL as all domains were affected in them (Table 3). 
Table 3 Comparison of HRQoL domains among PCOS Women by Demographic Characteristics

\begin{tabular}{|c|c|c|c|c|c|c|c|c|}
\hline \multirow[t]{2}{*}{ Demographics } & \multicolumn{4}{|l|}{ Emotions } & \multicolumn{4}{|c|}{ Body hairs } \\
\hline & n & Mean rank & Test stat & P value & n & Mean rank & Test stats & P value \\
\hline Organization & $\begin{array}{l}\text { Public }=114 \\
\text { Private }=38\end{array}$ & $\begin{array}{l}79.13 \\
68.61\end{array}$ & $1866.000^{\mathrm{a}}$ & 0.199 & $\begin{array}{l}114 \\
38\end{array}$ & $\begin{array}{l}75.88 \\
78.37\end{array}$ & $2095.000^{\mathrm{a}}$ & 0.767 \\
\hline Marital status & $\begin{array}{l}\text { Married }=77 \\
\text { Single }=70\end{array}$ & $\begin{array}{l}80.64 \\
66.70\end{array}$ & $2184.000^{\mathrm{a}}$ & $0.044^{b}$ & $\begin{array}{l}77 \\
70\end{array}$ & $\begin{array}{l}97.60 \\
48.04\end{array}$ & $877.500^{\mathrm{a}}$ & 0.001 \\
\hline setting & $\begin{array}{l}\text { Urban }=112 \\
\text { Rural }=40\end{array}$ & $\begin{array}{l}72.12 \\
88.76\end{array}$ & $1749.500^{a}$ & 0.043 & $\begin{array}{l}112 \\
40\end{array}$ & $\begin{array}{l}72.43 \\
87.90\end{array}$ & $1784.000^{\mathrm{a}}$ & 0.054 \\
\hline Age of patient & $\begin{array}{l}15-25=84 \\
26-35=51 \\
36-50=17\end{array}$ & $\begin{array}{l}70.96 \\
90.57 \\
61.65 \\
\end{array}$ & $8.492^{\mathrm{b}}$ & 0.013 & $\begin{array}{l}84 \\
51 \\
17 \\
\end{array}$ & $\begin{array}{l}60.64 \\
99.76 \\
85.09 \\
\end{array}$ & $25.943^{b}$ & 0.002 \\
\hline Level of education & $\begin{array}{l}\text { Illiterate }=6 \\
\text { Primary }=25 \\
\text { Secondary }=55 \\
\text { Bachelors }=49 \\
\text { Master }=16\end{array}$ & $\begin{array}{l}105.42 \\
102.30 \\
73.54 \\
67.44 \\
58.56\end{array}$ & $16.389^{b}$ & 0.001 & $\begin{array}{l}6 \\
25 \\
55 \\
49 \\
16\end{array}$ & $\begin{array}{l}100.75 \\
106.98 \\
71.02 \\
63.47 \\
73.81\end{array}$ & $19.350^{\mathrm{b}}$ & 0.002 \\
\hline No of children & $\begin{array}{l}1=10 \\
2=10 \\
3=11 \\
4=3 \\
>4=3\end{array}$ & $\begin{array}{l}22.65 \\
20.95 \\
21.00 \\
5.00 \\
7.00\end{array}$ & $10.583^{b}$ & 0.022 & $\begin{array}{l}10 \\
10 \\
11 \\
3 \\
3\end{array}$ & $\begin{array}{l}22.80 \\
21.00 \\
18.50 \\
9.83 \\
10.67\end{array}$ & $5.585^{\mathrm{b}}$ & 0.238 \\
\hline Income & $\begin{array}{l}\leq 15,000=46 \\
15001-25,000=32 \\
25,001-35,000=41 \\
35,001-50,000=26 \\
\geq 50,000=7\end{array}$ & $\begin{array}{l}90.29 \\
73.11 \\
68.90 \\
72.40 \\
61.07 \\
\end{array}$ & $7.029^{\mathrm{b}}$ & 0.132 & $\begin{array}{l}46 \\
32 \\
41 \\
26 \\
7\end{array}$ & $\begin{array}{l}88.79 \\
77.34 \\
69.07 \\
62.04 \\
89.07 \\
\end{array}$ & $8.188^{\mathrm{b}}$ & 0.084 \\
\hline Work status & $\begin{array}{l}\text { Employed }=33 \\
\text { House wife }=76 \\
\text { Studying }=43\end{array}$ & $\begin{array}{l}72.82 \\
81.89 \\
69.80\end{array}$ & $2.370^{\mathrm{b}}$ & 0.315 & $\begin{array}{l}33 \\
76 \\
43\end{array}$ & $\begin{array}{l}65.32 \\
96.52 \\
49.70\end{array}$ & $33.976^{\mathrm{b}}$ & 0.002 \\
\hline Type of comorbidity & $\begin{array}{l}\text { None }=134 \\
\text { Hypertension=9 } \\
\text { Other }=2\end{array}$ & $\begin{array}{l}72.26 \\
94.89 \\
24.25\end{array}$ & $5.192^{\mathrm{b}}$ & 0.062 & $\begin{array}{l}134 \\
9 \\
2\end{array}$ & $\begin{array}{l}71.93 \\
97.83 \\
32.75\end{array}$ & $5.097^{\mathrm{b}}$ & 0.071 \\
\hline Type of therapy & Hormonal= 51 & 81.91 & $1.165^{\mathrm{b}}$ & $0.567^{b}$ & 51 & 79.58 & $0.727^{\mathrm{b}}$ & 0.710 \\
\hline
\end{tabular}




\begin{tabular}{|l|l|l|l|l|l|l|l|l|}
\hline & Other=10 & 73.10 & & & 10 & 67.15 & & \\
& None=91 & 73.84 & & & 91 & 75.80 & \\
\hline Thyroid problem & Yes=3 & 84.83 & $198.500^{\mathrm{a}}$ & 0.759 & 3 & 102.00 & $147.000^{\mathrm{a}}$ & 0.332 \\
& No=149 & 76.33 & & & 149 & 75.99 & & \\
\hline Built of patient & Heavy=87 & 87.29 & $12.328^{\mathrm{b}}$ & $\mathbf{0 . 0 0 2}$ & 87 & 84.82 & $9.155^{\mathrm{b}}$ & $\mathbf{0 . 0 1 1}$ \\
& Normal=37 & 60.80 & & & 37 & 58.92 & & \\
& Slim=28 & 63.71 & & & 28 & 73.89 & & \\
\hline
\end{tabular}

Mann-Whitney Test $(p \geq 0.05)^{a}$, Krsukal Wallis Test $\left(p \geq 0.05^{b}\right)$.

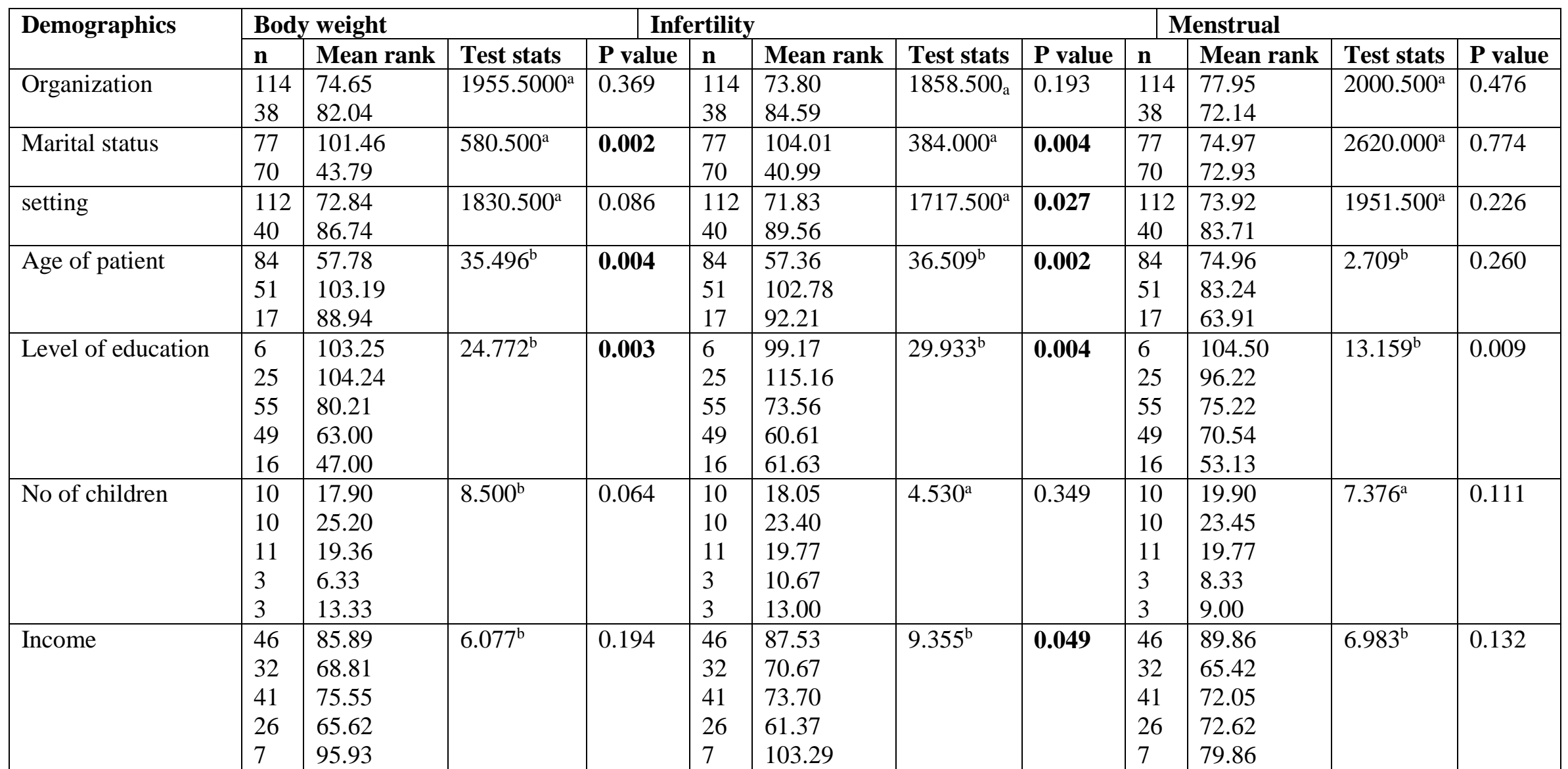




\begin{tabular}{|c|c|c|c|c|c|c|c|c|c|c|c|c|}
\hline Work status & $\begin{array}{l}33 \\
76 \\
43\end{array}$ & $\begin{array}{l}60.79 \\
98.74 \\
49.24\end{array}$ & $40.325^{\mathrm{b}}$ & 0.002 & $\begin{array}{l}33 \\
76 \\
43\end{array}$ & $\begin{array}{l}67.26 \\
96.13 \\
48.90\end{array}$ & $33.745^{\mathrm{b}}$ & 0.004 & $\begin{array}{l}33 \\
76 \\
43\end{array}$ & $\begin{array}{l}75.06 \\
79.04 \\
73.12\end{array}$ & $0.547^{\mathrm{a}}$ & 0.766 \\
\hline Type of comorbidity & $\begin{array}{l}134 \\
9 \\
2 \\
\end{array}$ & $\begin{array}{l}71.62 \\
99.00 \\
48.75\end{array}$ & $4.286^{\mathrm{b}}$ & 0.109 & $\begin{array}{l}134 \\
9 \\
2 \\
\end{array}$ & $\begin{array}{l}72.37 \\
94.44 \\
18.75\end{array}$ & $5.761^{\mathrm{b}}$ & 0.040 & $\begin{array}{l}134 \\
9 \\
2\end{array}$ & $\begin{array}{l}73.20 \\
80.28 \\
27.00\end{array}$ & $2.696^{\mathrm{b}}$ & 0.280 \\
\hline Type of therapy & $\begin{array}{l}51 \\
10 \\
91\end{array}$ & $\begin{array}{l}72.06 \\
97.25 \\
76.71 \\
\end{array}$ & $2.759^{\mathrm{ba}}$ & 0.252 & $\begin{array}{l}51 \\
10 \\
91\end{array}$ & $\begin{array}{l}77.10 \\
81.90 \\
75.57 \\
\end{array}$ & $0.202^{\mathrm{b}}$ & 0.915 & $\begin{array}{l}51 \\
10 \\
91\end{array}$ & $\begin{array}{l}74.70 \\
64.45 \\
78.84 \\
\end{array}$ & $1.100^{\mathrm{b}}$ & 0.589 \\
\hline Thyroid problem & $\begin{array}{l}3 \\
149 \\
\end{array}$ & $\begin{array}{l}128.67 \\
75.45\end{array}$ & $67.000^{\mathrm{a}}$ & 0.037 & $\begin{array}{l}3 \\
149 \\
\end{array}$ & $\begin{array}{l}112.67 \\
75.77 \\
\end{array}$ & $115.000^{\mathrm{a}}$ & 0.165 & $\begin{array}{l}3 \\
149\end{array}$ & $\begin{array}{l}82.00 \\
76.39 \\
\end{array}$ & $207.000^{\mathrm{a}}$ & 0.835 \\
\hline Built of patient & $\begin{array}{l}87 \\
37 \\
28\end{array}$ & $\begin{array}{l}91.77 \\
46.16 \\
69.14\end{array}$ & $28.991^{b}$ & 0.002 & $\begin{array}{l}87 \\
37 \\
28\end{array}$ & $\begin{array}{l}91.34 \\
53.26 \\
61.11\end{array}$ & $23.812^{\mathrm{a}}$ & 0.003 & $\begin{array}{l}87 \\
37 \\
28\end{array}$ & $\begin{array}{l}86.44 \\
62.30 \\
64.39\end{array}$ & $10.489^{\mathrm{a}}$ & 0.005 \\
\hline
\end{tabular}


The results highlighted that $42.1 \%(n=64)$ of the patients feel tense most of the time and $59.9 \%$ $(n=91)$ felt sort of frightened feeling very definitely and quite badly as if something awful is about to happen. Most patients having a great deal of the time worrying thoughts go through their mind $(n=114,75 \%)$ and $8.6 \%(n=13)$ did not feel cheerful often. On the other hand, $13.8 \%(\mathrm{n}=21)$ definitely lost interest in their appearance, $53.3 \%(\mathrm{n}=81)$ get panic very often and $24.3 \%(\mathrm{n}=37)$ not often enjoy a book or TV program (Table 4).

Table 4: Assessment of Depression among Polycystic Ovary Syndrome Women

\begin{tabular}{|c|c|c|}
\hline Indicators & & n (\%) \\
\hline \multirow[t]{4}{*}{ I feel tense or 'wound up'; } & Not at all & $1(0.7)$ \\
\hline & From time to time, occasionally & $42(27.6)$ \\
\hline & A lot of the time & $45(29.6)$ \\
\hline & Most of the time & $64(42.1)$ \\
\hline \multirow{4}{*}{$\begin{array}{l}\text { I still enjoy the things I used to } \\
\text { enjoy }\end{array}$} & Definitely as much & $54(35.5)$ \\
\hline & Not quite so much & $36(23.7)$ \\
\hline & Only a little & $35(23)$ \\
\hline & Hardly at all & $27(17.8)$ \\
\hline \multirow{4}{*}{$\begin{array}{l}\text { I get a sort of frightened feeling as } \\
\text { if something awful is about to } \\
\text { happen }\end{array}$} & Not at all & $23(15.1)$ \\
\hline & A little, but it doesn't worry me & $19(12.5)$ \\
\hline & Yes, but not too badly & $19(12.5)$ \\
\hline & Very definitely and quite badly & $91(59.9)$ \\
\hline \multirow{4}{*}{$\begin{array}{l}\text { I can laugh and see the funny side } \\
\text { of things }\end{array}$} & Not at all & $20(13.2)$ \\
\hline & Definitely not so much now & $30(19.7)$ \\
\hline & Not quite so much now & $42(27.6)$ \\
\hline & As much as I always could & $60(39.5)$ \\
\hline \multirow{4}{*}{$\begin{array}{l}\text { Worrying thoughts go through my } \\
\text { mind }\end{array}$} & Only occasionally & $6(3.9)$ \\
\hline & $\begin{array}{l}\text { From time to time, but not too often } \\
\text { A lot of the time }\end{array}$ & $18(11.8)$ \\
\hline & A great deal of the time & $14(9.2)$ \\
\hline & & $114(75)$ \\
\hline \multirow[t]{4}{*}{ I feel cheerful } & Most of the time & $35(23)$ \\
\hline & Sometimes & $61(40.1)$ \\
\hline & Not often & $43(28.3)$ \\
\hline & Not at all & $13(8.6)$ \\
\hline \multirow[t]{4}{*}{ I can sit at ease and feel relaxed } & Definitely & $13(8.6)$ \\
\hline & Usually & $72(47.4$ \\
\hline & Not Often & $58(38.2)$ \\
\hline & Not at all & $9(5.9)$ \\
\hline \multirow[t]{4}{*}{ I feel as if I am slowed down } & Not at all & $17(11.2)$ \\
\hline & Sometimes & $32(21.1)$ \\
\hline & Very often & $52(34.2)$ \\
\hline & Nearly all the time & $51(33.6)$ \\
\hline I get a sort of frightened feeling & Not at all & $24(15.8)$ \\
\hline \multirow[t]{3}{*}{ like 'butterflies' in the stomach } & Occasionally & $52(34.2)$ \\
\hline & Quite Often & $55(36.2)$ \\
\hline & Very Often & $21(13.8)$ \\
\hline \multirow{4}{*}{$\begin{array}{l}\text { I have lost interest in my } \\
\text { appearance }\end{array}$} & I take just as much care as ever & $56(36.8)$ \\
\hline & I may not take quite as much care & $25(16.4)$ \\
\hline & I don't take as much care as I should & $50(32.9)$ \\
\hline & Definitely & $21(13.8)$ \\
\hline
\end{tabular}




\begin{tabular}{lll}
\hline I feel restless as I have to be on the & Not at all & $6(3.9)$ \\
move & Not very much & $25(16.4)$ \\
& Quite a lot & $70(46.1)$ \\
& Very much indeed & $51(33.6)$ \\
I look forward with enjoyment to & As much as I ever did & $70(46.1)$ \\
things & Rather less than I used to & $30(19.7)$ \\
& Definitely less than I used to & $27(17.8)$ \\
& Hardly at all & $25(16.4)$ \\
I get sudden feelings of panic & Very often indeed & $81(53.3)$ \\
& Quite often & $40(26.3)$ \\
& Not very often & $27(17.8)$ \\
& Not at all & $4(2.6)$ \\
I can enjoy a good book or radio or & Often & $37(24.3)$ \\
& Sometimes & $38(25)$ \\
& Not often & $37(24.3)$ \\
& Very seldom & $40(26.3)$ \\
\hline
\end{tabular}

No significant difference $(\mathrm{p} \geq 0.05)$ in level of depression among PCOS women were observed in relation to different demographic variables including sector, marital status, setting, age, number of children, type of therapy, work status and those with thyroid issues. Significant difference $(p \geq 0.05)$ in level of depression among PCOS women were observed in relation to setting, qualification and income levels. Women with PCOS residing in rural areas were found relatively more depressed and those belonging to low educational and income background were comparatively found more depressed (Table 5).

Table 5 Comparison of Depression among Polycystic Ovary Syndrome Women by Demographic Characteristics

\begin{tabular}{|c|c|c|c|c|}
\hline Demographics & \multicolumn{4}{|l|}{ Composite score } \\
\hline \multirow[t]{2}{*}{ Sector } & Public $=114$ & 75.29 & $2028.500^{\mathrm{a}}$ & 0.567 \\
\hline & Private $=38$ & 80.12 & & \\
\hline \multirow[t]{2}{*}{ Marital status } & Married $=77$ & 71.87 & $2531.000^{\mathrm{a}}$ & 0.527 \\
\hline & Unmarried $=70$ & 76.34 & & \\
\hline \multirow[t]{2}{*}{ Setting } & Urban $=112$ & 72.76 & $1821.000^{\mathrm{a}}$ & 0.03 \\
\hline & Rural $=40$ & 86.98 & & \\
\hline \multirow[t]{3}{*}{ Age of patient } & $15-25=84$ & 72.05 & $3.596^{\mathrm{b}}$ & 0.166 \\
\hline & $26-35=51$ & 85.98 & & \\
\hline & $36-50=17$ & 70.03 & & \\
\hline \multirow[t]{5}{*}{ Level of education } & Illiterate $=6$ & 84.67 & $9.534^{\mathrm{b}}$ & 0.049 \\
\hline & Primary $=25$ & 96.80 & & \\
\hline & Secondary $=55$ & 71.67 & & \\
\hline & Bachelors = 49 & 75.45 & & \\
\hline & Master $=16$ & 56.81 & & \\
\hline \multirow[t]{5}{*}{ No of children } & One $=10$ & 22.65 & $7.515^{\mathrm{b}}$ & 0.111 \\
\hline & Two $=10$ & 21.30 & & \\
\hline & Three $=11$ & 19.41 & & \\
\hline & Four $=3$ & 9.50 & & \\
\hline & More than four $=3$ & 7.17 & & \\
\hline \multirow[t]{2}{*}{ Work status } & Employed = 33 & 70.42 & $1.972^{\mathrm{b}}$ & 0.373 \\
\hline & House wife $=76$ & 74.91 & & \\
\hline
\end{tabular}




\begin{tabular}{lllll}
\hline \multirow{3}{*}{ Income } & Studying $=43$ & 83.98 & & \\
& $\leq 15,000=46$ & 83.41 & $8.201^{\mathrm{b}}$ & $\mathbf{0 . 0 4 2}$ \\
& $15,001-25,000=32$ & 61.81 & & \\
Type of therapy & $35,001-35,000=41$ & 64.16 & & \\
& Hormonal $=51$ & 71.61 & $2.835^{\mathrm{b}}$ & 0.242 \\
& Other $=10$ & 97.10 & & \\
Thyroid problem & None $=91$ & 76.98 & & \\
& Yes $=3$ & 55.83 & $161.500^{\mathrm{a}}$ & 0.431 \\
& No $=149$ & 76.92 & & \\
\hline
\end{tabular}

Mann-Whitney Test ${ }^{a} ;$ Krsukal Wallis Test ${ }^{b}(p \geq 0.05)$

\section{DISCUSSION}

Polycystic ovary syndrome is one of the common endocrine disorder in adolescent ages which impact negatively on health related quality of life of women. The present study revealed impaired HRQoL among women with polycystic ovary syndrome. Most of them experienced lack of emotional control and mood stability. They also experienced aggressiveness due to PCOS. This might be due to the fact that prolonged physical treatment may lead towards mental and social discomfort. Similar results were reported from a study conducted in Germany highlighting mood disturbances such as irritation, impaired social and sexual and overall decreased life satisfaction among patients suffering from PCOS ${ }^{9}$.

PCOS can have an impact on social functioning due to stigma associated with it. Body dissatisfaction strongly associated to it also decrease quality of life ${ }^{16}$. The results of the present study showed patients often felt different from normal women. Most of them felt concerned about being overweight and the need to reduce weight. Moreover, weight reduction was a relatively taken as major concern among married women. Increased growth of body hairs was also considered an important issue in effecting overall quality of life, especially among married women. These finding are in accordance with a study conducted in Bulgaria which reported obesity as a significant feature of illness and had a negative impact on quality of life of PCOS women ${ }^{17}$. The results of the present study further reported infertility as a major factor effecting HRQoL. Similar findings of poor HRQoL has been reported among women having age $>25 \mathrm{yrs}$ due to infertility ${ }^{17}$.

In PCOS patients the actual reason for the depression is unknown and complex ${ }^{18}$. The results of the present study reported low HRQoL along with high levels of depression among PCOS women. Similar high prevalence of depression was found among PCOS women in India ${ }^{19}$. Furthermore, the present study reported that the patients known with thyroid problem and heavy built had poor HRQoL. These results are in line with different studies which also showed that high BMI and increased waist circumference are prone to depression and considered as high risk patient for PCOS ${ }^{20,21}$. 
Hirsutism associated with PCOS develops in later stage in life. The results of the present study revealed that body hairs, weight and infertility mostly affect HRQoL of house wives as compared to working women. It was interesting to notice that the concern for weight management, menstruation problems, hirutusim and infertility impacted relatively more on HRQoL among women residing in rural areas. They had poor emotional control and low HRQoL along with higher rates of depression. This might be due to the fact that they have less awareness, access and resources for better treatment and care. Similar finding from a study conducted in USA reported link of depression is associated with hirsutism and disturbed sleep among PCOS women ${ }^{3}$.

Higher QoL has been associated with higher education level ${ }^{22}$.The results of the present study revealed that illiterate or less educated women having PCOS had impaired HRQoL across all domains. Moreover, they were more depressed as compared to the educated patients. Similar findings were reported from a study conducted in USA highlighting that risk of PCOS decreases in patients who attained high level of education ${ }^{23}$. Income is considered another important factor contributing towards low HRQoL and high rates of depression among PCOS women. The results of present study showed that although, socioeconomic status had no impact on HRQoL of PCOS patients but do have an impact on depression. Although, HRQoL was low among women with different socioeconomic backgrounds but the ones with income less that Rs 15,000 were found relatively more depressed that the others. These findings are in accordance with a study conducted in USA which reported women who undergo low childhood socioeconomic status are at increased risk of polycystic ovary syndrome and higher depression rates in their life ${ }^{23}$.Furthermore, the present study revealed that comorbidity and different types of therapy does not affect any domain of HRQoL and depression. Physical activity, acupressure and to lesser extent resveratrol may improve psychological, hormonal and metabolic profile in women with PCOS ${ }^{24}$.

\section{CONCLUSION}

The results of the present study concluded that polycystic ovary syndrome had a negative impact on health related quality of life of patients across all domains with a significant likelihood of depression. While all domains were affected, the greatest impact was seen on infertility followed by body hairs and role limitation due to increased psychological and emotional problems. Low educational and income background had negative effect on mental and emotional HRQoL of polycystic ovary syndrome patients and depression. More attention should be given on improvement of health related quality of life among PCOS patients to decrease the rate of treatment failure and improve treatment response. Moreover, awareness programs targeting patients with low educational level should be initiated to increase health 
awareness related to impact of mental and emotional wellbeing on HRQoL among PCOS patients. All stakeholders need to work together for enhancement of physical and mental health related quality of life of PCOS patients in order to improve medication adherence, wellbeing and social functioning of PCOS patients.

\section{REFERENCES}

1. Behboodi Moghadam, Z., et al., Measures of health-related quality of life in PCOS women: a systematic review. 2018. Volume 10: p. 397-408.

2. Beiraghdar, M., et al., The relationship between quality of life and coping strategies in polycystic ovary syndrome patients. 2015. 4: p. 168.

3. Pehlivanov, B., et al., Determinants of health related quality of life in women with polycystic ovary syndrome. 2006. 45: p. 29-34.

4. Ali, S.S.M., A. Tariq, and A. Tariq. Anxiety and depression in patients with polycystic ovarian syndrome. in 20th European Congress of Endocrinology. 2018. BioScientifica.

5. Radhakrishnan, R. and A. Verghese, A Study On Anxiety And Depression Among Patients With Polycystic Ovary Syndrome. 2018. 8: p. 338-340.

6. Over, P., et al., Depression, Anxiety, and Anger in Patients with Polycystic Ovary Syndrome. 2014. 51: p. 328-333.

7. Greenwood, E.A., et al., Quality of life and depression in polycystic ovary syndrome. Fertility and sterility, 2017. 108(3): p. e64-e65.

8. Nasiri-Amiri, F., et al., The Polycystic Ovary Syndrome Health-Related Quality-ofLife Questionnaire: Confirmatory Factor Analysis. International journal of endocrinology and metabolism, 2018. 16(2).

9. Janssen, O., et al., Mood and Sexual Function in Polycystic Ovary Syndrome. 2008. 26: p. 45-52.

10. Amna, A., Hyperinsulinemia and Its Association With Poly Cystic Ovarian Syndrome. The Professional Medical Journal, 2014. 21(05): p. 946-949.

11. Ahmed, Y., et al., Polycystic ovarian syndrome: a new perspective. JPMA. The Journal of the Pakistan Medical Association, 2003. 53(2): p. 72-77.

12. Sadeeqa, S., T. Mustafa, and S. Latif, Polycystic ovarian syndrome-related depression in adolescent girls: A Review. Journal of Pharmacy And Bioallied Sciences, 2018. 10(2): p. 55.

13. Zehra, S., et al., Depression and anxiety in women with polycystic ovary syndrome from Pakistan. Life Sci J, 2015. 12(3s): p. 1-4.

14. Gul, S., S.A. Zahid, and A. Ansari, PCOS: symptoms and awareness in urban Pakistani women. Int J Pharma Res Health Sci, 2014. 2(5): p. 356-60. 
15. Rizvi, M., et al., Perception and attitude of patients regarding polycystic ovarian syndrome (PCOS) in tertiary care hospitals of Pakistan-a survey based study. International Journal of Pharmacy \& Therapeutics, 2014. 5(3): p. 147-152.

16. Pastore, L.M., et al., Depression symptoms and body dissatisfaction association among polycystic ovary syndrome women. Journal of psychosomatic research, 2011. 71(4): p. 270-276.

17. Mitkov, M., B. Pehlivanov, and D. Terzieva, Metformin versus rosiglitazone in the treatment of polycystic ovary syndrome. European Journal of Obstetrics \& Gynecology and Reproductive Biology, 2006. 126(1): p. 93-98.

18. Himelein, M.J. and S.S. Thatcher, Depression and body image among women with polycystic ovary syndrome. Journal of health psychology, 2006. 11(4): p. 613-625.

19. Upadhyaya, S.K., A. Sharma, and A. Agrawal, Prevalence of anxiety and depression in polycystic ovarian syndrome. Int J Med Sci Public Health, 2016. 5(4): p. 681-683.

20. Madhavan, R., Prevalence of PCOS diagnoses among women with menstrual irregularity in a diverse, multiethnic cohort. 2018, Boston University.

21. Mizgier, M., et al., Risk factors of overweight and obesity related to diet and disordered eating attitudes in adolescent girls with clinical features of polycystic ovary syndrome. Journal of Clinical Medicine, 2020. 9(9): p. 3041.

22. Milsom, S., et al., Polycystic Ovary Syndrome and Depression in New Zealand Adolescents. 2013. 26.

23. Merkin, S.S., et al., Socioeconomic status and polycystic ovary syndrome. Journal Of Women's Health, 2011. 20(3): p. 413-419.

24. Wardle, J., Dysmenorrhoea and menstrual complaints. Clinical Naturopathy: An evidence-based guide to practice, 2010: p. 346.

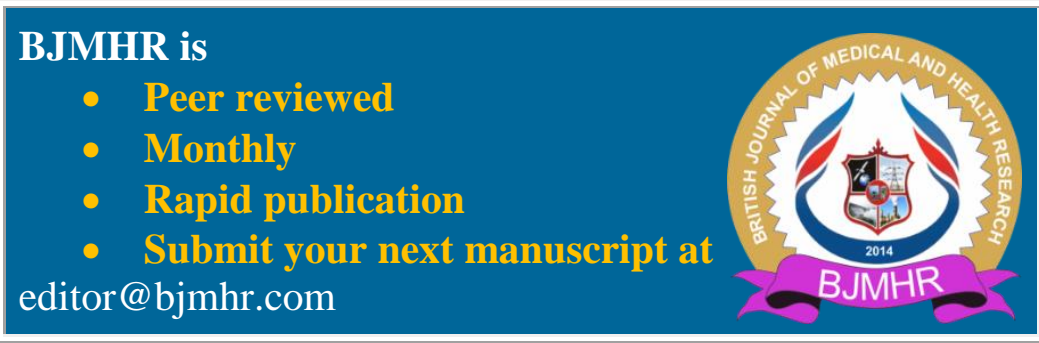

